\title{
A NOTE ON AKBULUT CORKS
}

\author{
Nikolai SAVELIEV
}

\begin{abstract}
We prove that the involution on the boundary $\Sigma$ of the Akbulut cork relating blown up elliptic surfaces to completely decomposable manifolds acts nontrivially on the Floer homology of $\Sigma$. We also show that $\Sigma$ provides an example of an irreducible manifold with non-zero boundary operator in its Floer chain complex.
\end{abstract}

Let $X_{0}$ and $X_{1}$ be smooth, closed, oriented, simply connected 4-manifolds. If $X_{0}$ is homeomorphic to $X_{1}$ then there is a compact contractible 4-manifold $W \subset X_{0}$ such that by cutting $W$ out of $X_{0}$ and re-gluing it by an involution on its boundary, we obtain a smooth 4-manifold diffeomorphic to $X_{1}$, see [6] and [12]. This contractible piece $W$ is called an Akbulut cork corresponding to the pair $\left(X_{0}, X_{1}\right)$.

Explicit examples of Akbulut corks were constructed in [1] and [11]. They correspond to pairs $\left(X_{0}(n), X_{1}(n)\right)$ where $X_{0}(n)=E(n) \#\left(-\mathbb{C} P^{2}\right)$ is a blow up of the elliptic surface $E(n)$, and $X_{1}(n)=(2 n-1) \cdot \mathbb{C} P^{2} \# 10 n \cdot\left(-\mathbb{C} P^{2}\right)$, with $n \geq 2$. For any given $n \geq 2$, the manifolds $X_{0}(n)$ and $X_{1}(n)$ are homeomorphic but not diffeomorphic. Their Akbulut cork $W$ is independent of $n$ and is obtained by attaching a two-handle to $S^{1} \times D^{3}$ along its boundary as shown in Figure 1 .

Observe that $\Sigma=\partial W$ is an integral homology sphere obtained by surgery on the link in Figure 1 both components of which are 0 -framed, and that it is symmetric with respect to the involution $\tau: \Sigma \rightarrow \Sigma$ interchanging the two link components. The manifold $X_{1}(n)$ is obtained from $X_{0}(n)$ by cutting out $W$ and re-gluing it using $\tau$.

The goal of this paper is to study the homomorphism $\tau_{*}: I_{*}(\Sigma) \rightarrow I_{*}(\Sigma)$ which $\tau$ induces on the Floer homology of $\Sigma$, see [9].

Theorem 1. Let $\Sigma$ be the boundary of manifold $W$ shown in Figure 1. Then

(1) $I_{n}(\Sigma)=0$ if $n$ is even, and $I_{n}(\Sigma)=\mathbb{Z}$ if $n$ is odd, and

(2) the homomorphism $\tau_{*}: I_{*}(\Sigma) \rightarrow I_{*}(\Sigma)$ is a non-trivial involution.

This result gives an insight into how re-gluing of $W$ leads to different smooth structures, via the study of the effect that $\tau_{*}$ has on the Donaldson invariants, see Section 4.

Received May 5, 2001.

Research was supported in part by NSF Grants DMS-9896376 and DMS-0196523 and MaxPlanck-Institut für Mathematik, Bonn. 
Our proof of Theorem 1 also implies the following result. As far as we know, this is the first example of an irreducible homology sphere having this property.

Theorem 2. The Floer chain complex of $\Sigma$ has a non-trivial boundary operator.

It should be mentioned that in general constructing diffeomorphisms acting non-trivially on the Floer homology is not an easy task. This is due in part to a close relation between such actions and exotic smooth structures on 4-manifolds. We briefly discuss these and related issues in Section 5 .

I am thankful to Selman Akbulut, whose papers [1] and [2] have motivated this research, and to Jim Bryan, Olivier Collin, Ronald Fintushel and Slawomir Kwasik for inspiring discussions concerning the matters in this paper. I am also thankful to the referee for pointing out an omission in the original argument. The Maple software package was used for calculations in Section 2.

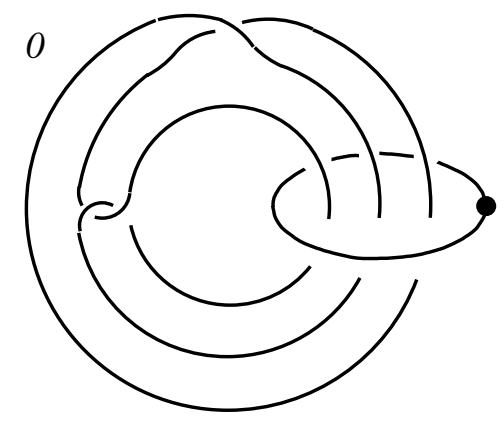

Figure 1

\section{Floer homology of $\Sigma$}

The Floer homology groups of $\Sigma$ are not difficult to compute by using the Floer exact triangle and Kirby calculus.

Proposition 3. The groups $I_{n}(\Sigma)$ vanish for even $n$, and $I_{n}(\Sigma)=\mathbb{Z}$ for odd $n$.

Proof. For an integer $p$, define an integral homology sphere $\Sigma_{p}$ as the boundary of a contractible 4-manifold obtained by surgery on the link shown in Figure 1 with the framing of the two-handle equal to $p$. Then $\Sigma_{0}=\Sigma$ and $\Sigma_{3}$ is orientation preserving diffeomorphic to the Brieskorn homology sphere $\Sigma(2,5,7)$, see [3]. According to [8], we have $I_{n}(\Sigma(2,5,7))=0$ for even $n$ and $I_{n}(\Sigma(2,5,7))=\mathbb{Z}$ for odd $n$. Therefore, the proposition will follow as soon as we prove that $I_{*}\left(\Sigma_{p}\right)$ is independent of $p$.

Let us perform a (-1)-surgery along the unknot $S$ shown in Figure 2. This surgery results in replacing framing $p$ by framing $p+1$ while preserving the rest of the picture. On the other hand, 0-surgery along the same circle yields the 


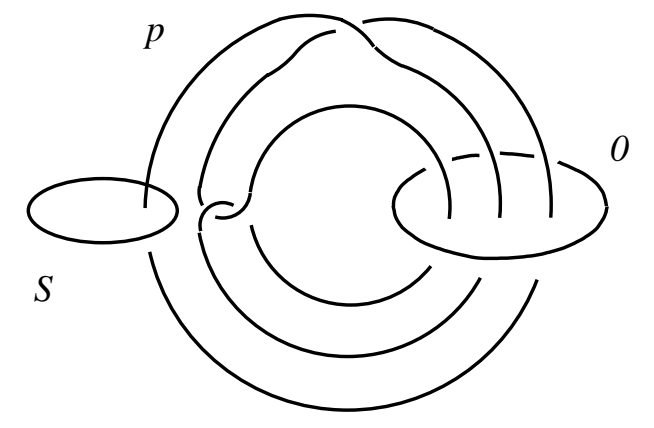

Figure 2

manifold $\bar{K}=S^{1} \times S^{2}$ with the trivial Floer homology, $I_{*}(\bar{K})=0$. The Floer exact triangle [10]

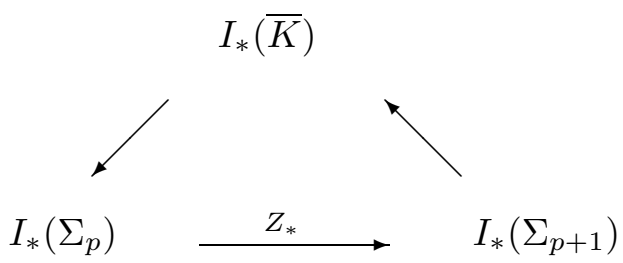

now implies that the trace $Z$ of the $(-1)$-surgery induces a degree zero isomorphism in Floer homology. This proves that $I_{*}\left(\Sigma_{p}\right)$ is independent of $p$.

The homomorphism $\tau_{*}: I_{*}(\Sigma) \rightarrow I_{*}(\Sigma)$ induced by the involution $\tau$ has degree zero. The fact that $\tau^{2}=1$ implies that $\left(\tau_{*}\right)^{2}=1$; therefore, on each of the factors $\mathbb{Z}$, the homomorphism $\tau_{*}$ is either identity or minus identity. A careful analysis of the Floer chain complex of $\Sigma$ in the next section will help us sort this out.

\section{The representation variety of $\pi_{1} \Sigma$}

After due simplifications, the standard Wirtinger presentation of the fundamental group of $\Sigma$ is of the form

$$
\pi_{1} \Sigma=\left\langle a, b \mid(b a)^{2}(a b)^{-2} b^{-1}(a b)^{2}=a^{2},(a b)^{2}(b a)^{-2} a^{-1}(b a)^{2}=b^{2}\right\rangle
$$

where $a$ and $b$ are meridians of the two components of the link in Figure 1 exchanged by the involution $\tau$. Specifying a representation $\alpha: \pi_{1} \Sigma \rightarrow S U(2)$ amounts to specifying two $S U(2)$-matrices, $A=\alpha(a)$ and $B=\alpha(b)$, satisfying the above relations. Since $\Sigma$ is an integral homology sphere, its only reducible representation is the trivial one. We will assume therefore that $\alpha$ is irreducible, i.e. $A$ and $B$ do not commute. Conjugating if necessary, we may assume that

$$
A=\left(\begin{array}{cc}
t+i \sqrt{1-t^{2}} & 0 \\
0 & t-i \sqrt{1-t^{2}}
\end{array}\right) \quad \text { and }
$$




$$
B=\left(\begin{array}{cc}
u+i r \sqrt{1-u^{2}} & \sqrt{\left(1-r^{2}\right)\left(1-u^{2}\right)} \\
-\sqrt{\left(1-r^{2}\right)\left(1-u^{2}\right)} & u-i r \sqrt{1-u^{2}}
\end{array}\right)
$$

for some real $t, u$ and $r$ such that $-1<t, u, r<1$. The relations on the matrices $A$ and $B$ can be rewritten as

$$
(A B)^{-2} B(A B)^{2}=A^{-2}(B A)^{2} \quad \text { and } \quad(B A)^{-2} A(B A)^{2}=B^{-2}(A B)^{2} .
$$

From this we conclude that $\operatorname{tr} B=\operatorname{tr}\left(A^{-1} B A \cdot B\right)$ and $\operatorname{tr} A=\operatorname{tr}\left(B^{-1} A B \cdot A\right)$ and, after simplification, that

$$
2\left(u^{2}+r^{2}-u^{2} r^{2}\right)(t+1)=1 \text { and } 2\left(t^{2}+r^{2}-t^{2} r^{2}\right)(u+1)=1 .
$$

Solutions of these equations are of two types, $t+u=1 / 2$ and $t=u$. In terms of matrices $A$ and $B$, these two types correspond to $\operatorname{tr} A+\operatorname{tr} B=1$ and $\operatorname{tr} A=\operatorname{tr} B$.

Representations with $\operatorname{tr} A+\operatorname{tr} B=1$ can be found in a completely explicit form by solving the rest of the equations (1). There are two such representations, one with $\operatorname{tr} A=(1-\sqrt{5}) / 2$ and $\operatorname{tr} B=(1+\sqrt{5}) / 2$, and the other with $\operatorname{tr} A=$ $(1+\sqrt{5}) / 2$ and $\operatorname{tr} B=(1-\sqrt{5}) / 2$. The parameter $r$ for both representations equals $1 / \sqrt{5}$. We call these representations $\beta_{1}$ and $\beta_{2}$, respectively. They are permuted by the involution $\tau^{*}$ and, in particular, they have the same Floer index.

Since $\operatorname{tr} A=\operatorname{tr} B$ for the representations of the second type, the matrix $B$ is conjugate to $A$ and hence can be written as $B=U A U^{-1}$ for some $S U(2)$-matrix $U$ with $U^{2}=-I$; one may assume that

$$
U=\left(\begin{array}{cc}
i \rho & \sqrt{1-\rho^{2}} \\
-\sqrt{1-\rho^{2}} & -i \rho
\end{array}\right)
$$

with $0<\rho<1$. Equations (1) then reduce to the single equation

$$
(U A)^{4}(A U)^{-4} U^{-1} A^{-1} U(A U)^{4}=A^{2},
$$

which is equivalent to a system of three polynomial equations in $t$ and $\rho$. These equations have four solutions corresponding to the following values of $t$ and $\rho$. The parameter $2 t$ is a real solution of the equation

$$
z^{7}+z^{6}-5 z^{5}-6 z^{4}+6 z^{3}+5 z^{2}-2 z-1=0 .
$$

This equation has five real solutions but only four of them lie between -2 and 2. Every $t$ uniquely determines $\rho$ by the formula

$$
\rho=(1 / 5) \sqrt{19-68 t-212 t^{2}+600 t^{3}+1024 t^{4}-384 t^{5}-832 t^{6}} .
$$

We name these four representations $\alpha_{1}$ through $\alpha_{4}$. They are preserved by the involution $\tau^{*}$.

Proposition 4. The $S U(2)-$ representation variety of $\pi_{1} \Sigma$ consists of a trivial representation $\theta$ and six non-degenerate irreducible representations, $\alpha_{1}, \alpha_{2}, \alpha_{3}$, $\alpha_{4}, \beta_{1}$ and $\beta_{2}$. The involution induced by $\tau$ keeps the representations $\theta$ and $\alpha_{1}$, $\alpha_{2}, \alpha_{3}, \alpha_{4}$ fixed, and permutes $\beta_{1}$ and $\beta_{2}$. 
Proof. We only need to check that the representations are non-degenerate, that is, the group cohomology $H_{\gamma}^{1}\left(\pi_{1} \Sigma, \mathfrak{s u}(2)\right)$ with coefficients in the adjoint representation vanishes for all $\gamma=\alpha_{1}, \alpha_{2}, \alpha_{3}, \alpha_{4}, \beta_{1}$ and $\beta_{2}$. This can be seen as follows.

The dimension of the space of 1 -coboundaries equals the rank of the operator

$$
\left(\begin{array}{l}
I-\operatorname{Ad}_{A} \\
I-\operatorname{Ad}_{B}
\end{array}\right): \mathbb{R}^{3} \rightarrow \mathbb{R}^{6}
$$

where $I$ is the identity matrix and $\operatorname{Ad}_{A}(x)=A x A^{-1}$. Let us consider the threeby-three minor of (4) consisting of the rows with numbers 3,4 , and 6 . A direct calculations shows that its determinant equals

$$
8 t\left(1-r^{2}\right)\left(1-u^{2}\right) \sqrt{1-t^{2}}
$$

Since $t \neq 0$ for any of the representations $\gamma$, this determinant is not zero, and hence the rank of (4) is three.

The cocycles are crossed homomorphisms $\xi: \pi_{1} \Sigma \rightarrow \mathfrak{s u}(2)$, which can be identified with vectors $\left(\xi_{A}, \xi_{B}\right) \in \mathbb{R}^{6}$ satisfying the linear system of equations

$$
\left\{\begin{array}{l}
U_{A} \xi_{A}+U_{B} \xi_{B}=0 \\
V_{A} \xi_{A}+V_{B} \xi_{B}=0
\end{array}\right.
$$

arising from the two relations in $\pi_{1} \Sigma$. Here, $U_{A}, U_{B}, V_{A}$, and $V_{B}$ are threeby-three matrices which can be explicitly written in terms of $t, u$, and $r$. The dimension of the space of cocycles thus equals the co-rank of the block matrix

$$
X=\left(\begin{array}{ll}
U_{A} & U_{B} \\
V_{A} & V_{B}
\end{array}\right)
$$

For the representation $\beta_{1}$, consider the three-by-three minor of (5) at the intersection of the rows and columns numbered 1,2, and 4. A direct calculation shows that it equals the matrix

$$
\left(\begin{array}{rrr}
\sqrt{5} / 5 & -1+2 \sqrt{5} / 5 & 1+\sqrt{5} / 5 \\
1+2 \sqrt{5} / 5 & -5 / 4+11 \sqrt{5} / 20 & 1+2 \sqrt{5} / 5 \\
2-6 \sqrt{5} / 5 & 1 / 2+\sqrt{5} / 10 & -1-4 \sqrt{5} / 5
\end{array}\right)
$$

with determinant $(15-5 \sqrt{5}) / 4$. Therefore, the cocycles at $\beta_{1}$ have dimension three, so that $\beta_{1}$ is non-degenerate. An argument for $\beta_{2}$ is similar.

If $\gamma$ is one of the representations $\alpha$, it is more convenient to work with parameters $t$ and $\rho$. We consider the minor of (5) at the intersection of rows 1,2 , and 4 , and columns 1,2 , and 5 . Its determinant is a polynomial in $t$ and $\rho$, where $t$ and $\rho$ satisfy equations (2) and (3). Setting the determinant equal to zero gives us three polynomial equations, which do not have common solutions. Therefore, the co-rank of (5) in this case is also three, and all the $\alpha_{i}$ are non-degenerate.

Corollary. There exist non-zero boundary operators in the Floer chain complex of $\Sigma$. 
Proof. Since the representation variety of $\Sigma$ is non-degenerate, its Floer chain complex $I C_{*}(\Sigma)$ is generated by the six irreducible representations. Since only four Floer homology groups are non-trivial, and each of them is isomorphic to $\mathbb{Z}$, not all boundary operators in the chain complex vanish. The same conclusion can be drawn even more easily from the fact that $I C_{*}(\Sigma)$ has two generators, $\beta_{1}$ and $\beta_{2}$, of the same Floer index.

\section{The boundary operators}

The boundary operators in the Floer chain complex of $\Sigma$ deserve a closer attention.

Let $\mu$ denote the Floer index. From the knowledge of the Floer homology groups $I_{*}(\Sigma)$ and the representation variety of $\pi_{1} \Sigma$ we conclude that $\mu\left(\beta_{1}\right)=$ $\mu\left(\beta_{2}\right)=1 \bmod 2$ (keeping in mind that $\beta_{1}$ and $\beta_{2}$ are permuted by the operator $\tau_{*}$ which preserves Floer index) and that exactly one of the representations $\alpha_{1}$ through $\alpha_{4}$ has even Floer index; let us call it $\alpha$.

By an obvious dimensional count, the only non-zero incidence numbers can be those between $\alpha$ and $\beta_{1}$ and between $\alpha$ and $\beta_{2}$. These are determined by signed counts $\# \hat{\mathcal{M}}_{g}\left(\alpha, \beta_{1}\right)$ and $\# \hat{\mathcal{M}}_{g}\left(\alpha, \beta_{2}\right)$ of isolated points in the instanton moduli spaces, where $g$ is a generic metric on $\Sigma$. Due to the lack of equivariant transversality, in general, it is not clear if one can choose a generic metric $g$ so that $\tau$ is an isometry. Therefore, we cannot claim that $\tau$ identifies $\hat{\mathcal{M}}_{g}\left(\alpha, \beta_{1}\right)$ with $\hat{\mathcal{M}}_{g}\left(\alpha, \beta_{2}\right)$; however, the following weaker result will suffice for our purposes.

Proposition 5. Let $g$ be a generic metric then $\# \hat{\mathcal{M}}_{g}\left(\alpha, \beta_{1}\right)=\# \hat{\mathcal{M}}_{g}\left(\alpha, \beta_{2}\right)$.

Proof. We begin by showing that the signed counts $\# \hat{\mathcal{M}}_{g}\left(\alpha, \beta_{i}\right), i=1,2$, are independent of the choice of generic metric $g$. Given two such metrics, $g_{0}$ and $g_{1}$, consider the product cobordism $W=\Sigma \times I$ with metric extending $g_{0}$ and $g_{1}$ at the two boundary components. This cobordism induces a degree zero chain map

$$
W_{*}: I C_{*}\left(\Sigma, g_{0}\right) \rightarrow I C_{*}\left(\Sigma, g_{1}\right) .
$$

An easy calculation shows that $W_{*}(\alpha)=\# \mathcal{M}_{W}(\alpha, \alpha) \cdot \alpha=\alpha$, since the only isolated instanton in $\mathcal{M}_{W}(\alpha, \alpha)$ is flat. Similarly, $W_{*}\left(\beta_{1}\right)=\# \mathcal{M}_{W}\left(\beta_{1}, \beta_{1}\right)$. $\beta_{1}+\# \mathcal{M}_{W}\left(\beta_{1}, \beta_{2}\right) \cdot \beta_{2}=\beta_{1}$ and $W_{*}\left(\beta_{2}\right)=\beta_{2}$. The last two formulas use the observation that all isolated instantons in $\mathcal{M}_{W}\left(\beta_{1}, \beta_{2}\right)$ and $\mathcal{M}_{W}\left(\beta_{2}, \beta_{1}\right)$ are flat, which is due to the fact that the Chern-Simons functional takes the same value on both $\beta_{1}$ and $\beta_{2}$. However, there are no flat connections on $W$ interpolating between $\beta_{1} \neq \beta_{2}$. The fact that $W_{*}$ is a chain map now easily implies that $\# \hat{\mathcal{M}}_{g_{0}}\left(\alpha, \beta_{i}\right)=\# \hat{\mathcal{M}}_{g_{1}}\left(\alpha, \beta_{i}\right)$ for $i=1,2$.

To finish the proof, choose a generic metric $g$ on $\Sigma$. The metric $\tau_{*} g$ is then also generic, and we have a natural bijective correspondence $\hat{\mathcal{M}}_{g}\left(\alpha, \beta_{1}\right)=$ $\hat{\mathcal{M}}_{\tau_{*} g}\left(\alpha, \beta_{2}\right)$. This correspondence is orientation preserving, since $\tau$ preserves 
both orientation and homology orientation. Therefore,

$$
\# \hat{\mathcal{M}}_{g}\left(\alpha, \beta_{1}\right)=\# \hat{\mathcal{M}}_{\tau_{*} g}\left(\alpha, \beta_{2}\right)=\# \hat{\mathcal{M}}_{g}\left(\alpha, \beta_{2}\right)
$$

Since there is no torsion in $I_{*}(\Sigma)$, the above proposition implies that $\# \hat{\mathcal{M}}_{g}\left(\alpha, \beta_{1}\right)=\# \hat{\mathcal{M}}_{g}\left(\alpha, \beta_{2}\right)= \pm 1$. If $n=\mu\left(\beta_{1}\right)=\mu\left(\beta_{2}\right)$ then $I_{n}(\Sigma)=\mathbb{Z}$ is generated by $\beta_{1}$, and $\tau_{*}: I_{n}(\Sigma) \rightarrow I_{n}(\Sigma)$ is minus identity.

\section{Gluing formulas and equivariant Floer homology}

Let $X$ be a smooth closed simply connected 4-manifold split as $X=U \cup V$ with $U$ and $V$ smooth compact simply connected 4-manifolds such that $\partial U=\Sigma$ and $\partial V=-\Sigma$, where $\Sigma$ is an integral homology 3-sphere. Let $D(X)$ be a degree $d$ Donaldson polynomial corresponding to a bundle $P$ over $X$, and let $u_{1}, \ldots, u_{r} \in H_{2}(U, \mathbb{Z})$ and $v_{1}, \ldots, v_{d-r} \in H_{2}(V, \mathbb{Z})$. In favorable circumstances, there exist well-defined relative Donaldson polynomials $D(U)\left(u_{1}, \ldots, u_{r}\right)$ and $D(V)\left(v_{1}, \ldots, v_{d-r}\right)$ with coefficients in equivariant Floer homology $I_{*}^{\mathcal{G}}(\Sigma)$ and $I_{*}^{\mathcal{G}}(-\Sigma)$, respectively, such that $D(X)\left(u_{1}, \ldots, u_{r}, v_{1}, \ldots, v_{d-r}\right)$ is obtained from them by pairing $I_{*}^{\mathcal{G}}(\Sigma)$ with $I_{*}^{\mathcal{G}}(-\Sigma)$, see [4].

The equivariant Floer homology $I_{*}^{\mathcal{G}}(\Sigma)$ of Austin and Braam [4] is the homology of a chain complex built from all representations of $\pi_{1} \Sigma$, including reducible. When the representation variety of $\Sigma$ is non-degenerate, which is the case for $\Sigma=\partial W$, the boundary of the Akbulut cork $W$, this equivariant Floer homology is roughly described as follows. The group $S O(3)$ acts by conjugation on the representation space $R(\Sigma)=\operatorname{Hom}\left(\pi_{1} \Sigma, S U(2)\right)$ with quotient the representation variety in Proposition 4 . Let us consider the equivariant cohomology

$$
H_{S O(3)}^{*}(R(\Sigma), \mathbb{Z})=H^{*}\left(E S O(3) \times_{S O(3)} R(\Sigma), \mathbb{Z}\right)
$$

where $\operatorname{ESO}(3) \rightarrow B S O(3)$ is the universal $S O(3)$-bundle. The connected components of $R(\Sigma)$ correspond to the points in the representation variety of $\Sigma$. Let $R_{\alpha}$ be the component containing a representation $\alpha$. If $\alpha$ is irreducible, we have $R_{\alpha}=S O(3)$ and

$$
H_{S O(3)}^{*}\left(R_{\alpha}, \mathbb{Z}\right)=H^{*}\left(R_{\alpha} / S O(3), \mathbb{Z}\right)=\mathbb{Z}
$$

The component $R_{\theta}$ is a point so that $H_{S O(3)}^{*}\left(R_{\theta}, \mathbb{Z}\right)=H^{*}(B S O(3), \mathbb{Z})$. These cohomology groups together form the $E_{1}$ term of a Morse-Bott spectral sequence which converges to $I_{*}^{\mathcal{G}}(\Sigma)$, see [4].

If the intersection forms of both $U$ and $V$ have maximal positive subspaces of dimensions $b_{+}(U)>0$ and $b_{+}(V)>0$, the instantons restricting to reducible flat connections on $\Sigma$ can be perturbed away, and the regular Floer homology $I_{*}(\Sigma)$ can be used in the gluing formula instead of $I_{*}^{\mathcal{G}}(\Sigma)$.

Let now $W$ be the Akbulut cork shown in Figure 1, and consider the splittings

$$
X_{0}(n)=W \cup_{\text {id }} Q \quad \text { and } \quad X_{1}(n)=W \cup_{\tau} Q
$$


where $Q=X_{0}(n) \backslash$ int $W$ is a smooth compact simply connected 4-manifold with boundary $-\Sigma$. The manifold $W$ is contractible so that $b_{+}(W)=0$. An attempt to use $I_{*}(\Sigma)$ instead of $I_{*}^{\mathcal{G}}(\Sigma)$ in the gluing formula leads to a contradiction as follows.

The only relative Donaldson polynomial of $W$ has degree zero and an easy index calculation shows that $D(W) \in I_{5}(\Sigma)=\mathbb{Z}$. Since $X_{0}(n)$ is an algebraic surface, there exist homology classes $v_{1}, \ldots, v_{d} \in H_{2}(Q, \mathbb{Z})=H_{2}\left(X_{0}(n), \mathbb{Z}\right)$ such that $D\left(X_{0}(n)\right)\left(v_{1}, \ldots, v_{d}\right) \neq 0$, see [7]. Then

$$
D\left(X_{0}(n)\right)\left(v_{1}, \ldots, v_{d}\right)=D(W) \cdot D(Q)\left(v_{1}, \ldots, v_{d}\right) \neq 0
$$

is a product of two non-zero numbers, and the involution $\tau_{*}: I_{5}(\Sigma) \rightarrow I_{5}(\Sigma)$ can only change sign of this product. On the other hand, we know that re-gluing by $\tau$ makes $X_{0}(n)$ into $X_{1}(n)$. Since $X_{1}(n)$ is completely decomposable, all its Donaldson polynomials vanish.

This contradiction shows that there exist instantons on $X_{0}(n)$ which do not factor through irreducible flat connections on $\Sigma$, and the full group $I_{*}^{\mathcal{G}}(\Sigma)$ should be taken into account. This last remark clarifies the statement of [2].

\section{Concluding remarks}

The fact that the action $\tau_{*}: I_{*}(\Sigma) \rightarrow I_{*}(\Sigma)$ is non-trivial implies that the mapping cylinder $C_{\tau}$ of the involution $\tau$ is not diffeomorphic to the product $\Sigma \times I$ rel boundary (although manifolds $C_{\tau}$ and $\Sigma \times I$ are in fact diffeomorphic).

Proposition 6. The manifolds $C_{\tau}$ and $\Sigma \times I$ are not homeomorphic (or homotopy equivalent) rel boundary.

Proof. Let us identify the two boundary components of each of $C_{\tau}$ and $\Sigma \times I$ by using identity maps. We end up with closed manifolds $M_{\tau}$, which is the mapping torus of $\tau$, and $\Sigma \times S^{1}$, respectively. If $C_{\tau}$ and $\Sigma \times I$ were homeomorphic (or homotopy equivalent) rel boundary, we would have that $\pi_{1}\left(M_{\tau}\right)=\pi_{1}\left(\Sigma \times S^{1}\right)$. However, the latter is not the case, which can be seen as follows.

The fundamental groups of both $\Sigma \times S^{1}$ and $M_{\tau}$ are HNN-extensions of $\pi_{1}(\Sigma)$. This easily implies that irreducible $S U(2)$-representations of $\pi_{1}(\Sigma \times$ $\left.S^{1}\right)$, respectively, $\pi_{1}\left(M_{\tau}\right)$, are in two-to-one correspondence with irreducible $S U(2)$-representations of $\pi_{1}(\Sigma)$, respectively, irreducible $S U(2)$-representations of $\pi_{1}(\Sigma)$ equivariant with respect to $\tau$. Since there exist irreducible $S U(2)-$ representations of $\pi_{1}(\Sigma)$ which are not $\tau$-equivariant, we conclude that $\pi_{1}\left(M_{\tau}\right) \neq$ $\pi_{1}\left(\Sigma \times S^{1}\right)$.

The above proposition shows that the mapping cylinder of $\tau$ fails to give an example of an exotic smooth structure on $\Sigma \times I$. No such examples are currently known, although exotic smooth structures do exist on all non-compact manifolds $M \times \mathbb{R}$ where $M$ is a closed oriented 3-manifold, see [5].

Any orientation preserving diffeomorphism $f: \Sigma \rightarrow \Sigma$ of an integral homology sphere $\Sigma$ induces an automorphism $f_{*}: I_{*}(\Sigma) \rightarrow I_{*}(\Sigma)$ in its Floer homology. This automorphism is often an identity. Theorem 1 gives an example 
of $\tau: \Sigma \rightarrow \Sigma$ with $\tau_{*} \neq$ id; the manifold $\Sigma$ is in fact hyperbolic. Examples of irreducible graph homology spheres with involutions acting non-trivially on their Floer homology can be found in [13] and [14].

\section{References}

[1] S. Akbulut, A fake compact contractible 4-manifold, J. Diff. Geom. 33 (1991), 335-356.

[2] __ An involution permuting Floer homology, Turkish J. Math. 18 (1994), 16-22.

[3] S. Akbulut, R. Kirby, Mazur manifolds, Michigan Math. J. 26 (1979), 259-284.

[4] D. Austin, P. Braam, Equivariant Floer theory and gluing Donaldson polynomials, Topology 35 (1996), 167-200.

[5] Ž. Bižaca, J. Etnyre, Smooth structures on collarable ends of 4-manifolds, Topology 37 (1998), 461-467.

[6] C. Curtis, M. Freedman, W. Hsiang, R. Stong, A decomposition theorem for $h$-cobordant smooth simply-connected compact 4-manifolds, Inv. Math. 123 (1996), 343-348.

[7] S. Donaldson, P. Kronheimer, The geometry of four-manifolds, Oxford Univ. Press, 1990

[8] R. Fintushel, R. Stern, Instanton homology of Seifert fibred homology three spheres. Proc. London Math. Soc. 61 (1990), 109-137

[9] A. Floer, An instanton-invariant for 3-manifolds, Comm. Math. Phys. 118 (1988), 215240.

[10] Instanton homology, surgery, and knots, Geometry of low-dimensional manifolds, I (Durham, 1989), 97-114, LMS Note Ser., 150, Cambridge Univ. Press, Cambridge, 1990.

[11] R. Gompf, A. Stipsicz, 4-Manifolds and Kirby Calculus, AMS Graduate Studies in Mathematics, 20, Providence, 1999.

[12] R. Matveyev, A decomposition of smooth simply-connected h-cobordant 4-manifolds, J. Diff. Geom. 44 (1996), 571-582.

[13] D. Ruberman, N. Saveliev, Rohlin's invariant and gauge theory, II. Mapping tori, Preprint, 2003. http://arXiv.org/math.GT/0306188.

[14] N. Saveliev, An involution permuting Floer homology, J. Knot Theory 9 (2000), 543-556.

Department of Mathematics, University of Miami, PO Box 249085, FL 33124

E-mail address: saveliev@math.miami.edu 